\title{
Consumo alimentar residual e sua relação com medidas de desempenho e eficiência e características in vivo da carcaça de cordeiros
}

\author{
[The residual feed intake and its relationship with performance and efficiency measures and \\ in vivo carcass characteristics of lambs] \\ E.F.E. Paula ${ }^{1}$, A.L.G. Monteiro ${ }^{2}$, D.F. Souza ${ }^{1}$, O.R. Prado ${ }^{1}$, T.M. Nomura ${ }^{1}$, \\ T.S.S. Stivari ${ }^{1}$, C.J.A. Silva ${ }^{1}$, M.H.A. Santana ${ }^{3}$ \\ ${ }^{1}$ Pós-graduação - Universidade Federal do Paraná - UFPR - Curitiba, PR \\ ${ }^{2}$ Departamento de Zootecnia - Universidade Federal do Paraná - UFPR - Curitiba, PR \\ ${ }^{3}$ Pós-graduação - Universidade de São Paulo - USP/FAZEA - Pirassununga, SP
}

\begin{abstract}
RESUMO
Avaliou-se o consumo alimentar residual (CAR) e a conversão alimentar (CA) de 20 cordeiros, com o objetivo de estimar as correlações entre essas variáveis com medidas de desempenho e com características in vivo da carcaça. Os animais tiveram o consumo de MS (CMS) mensurado por 65 dias e foram pesados a cada 13 dias para obtenção do ganho médio diário (GMD). Foram considerados o peso vivo inicial (PVI), o peso vivo final (PVF), o peso metabólico (PM), o GMD, a taxa de crescimento relativo (TCR), a taxa de Kleiber (TK), a CA, o CMS e o CMS em percentual do PV (CMSPV). As avaliações de carcaça foram realizadas por ultrassom. O CAR se mostrou correlacionado com o CMS $(+0,81)$, o CMSPV $(+0,90)$ e a CA $(+0,63)$. Correlações significativas foram encontradas entre CA e GMD; CA e TCR; CA e TK; e CA e PVI (-0,63, -0,74, -0,75 e +0,51, respectivamente). O CAR e a CA não se mostraram correlacionados com características de carcaça, e, da mesma forma, não houve diferença entre as classes de CAR para essas variáveis. Confirmou-se o potencial do CAR como medida de eficiência alimentar para cordeiros em confinamento, sem existência de relações com o ganho de peso e o tamanho corporal e sem alterações na composição da carcaça.
\end{abstract}

Palavras-chave: deposição de gordura, eficiência alimentar, Ile de France, ovinos, ultrassonografia

\begin{abstract}
The residual feed intake (RFI) and the feed conversion ratio (FCR) of 20 lambs was evaluated in order to estimate the correlations between these traits and performance measures and with in vivo carcass characteristics. The animals had their dry matter intake (DMI) measured over 65 days and they were weighed every 13 days to determine the average daily weight gain (ADG). Initial body weight (IBW), final body weight $(F B W)$, metabolic weight $(M W), A D G$, relative growth rate $(R G R)$, Kleiber ratio $(K R)$, $F C R, D M I$ and DMI in BW percentual (BWDMI) were considered. The carcass evaluation was performed by ultrasound. The RFI was correlated with the DMI (+0.81), BWDMI (+0.90) and with FCR (+0.63). Significant correlations were found between FCR and ADG; FCR and RGR; and FCR and KR; and FCR and $I B W(-0.63,-0.74,-0.75$ and +0.51 respectively). The RFI and the FCR were not correlated with carcass traits and similarly there was no difference between the RFI-classes for these variables. It was proved the RFI has potential as a measure of feed efficiency for housed lambs, without the existence of relations with weight gain and body size of animals and without changes in carcass composition.
\end{abstract}

Keywords: fat deposition, feed efficiency, Ile de France, sheep, ultrasonography

Recebido em 1 de março de 2012

Aceito em 6 de novembro de 2012

E-mail: efevaristo@globo.com 


\section{INTRODUÇÃO}

A ovinocultura tem se consolidado cada vez mais no cenário da pecuária mundial. Segundo dados da FAO (Food and Agriculture Organization, 2009), o rebanho efetivo de ovinos já ultrapassa a faixa de 1 bilhão de indivíduos e o interesse por essa atividade é visivelmente crescente. Dessa maneira, torna-se imperiosa a busca pela identificação e seleção de animais que utilizem mais eficientemente o alimento que lhes é fornecido, uma vez que o melhor aproveitamento da dieta acarreta importantes benefícios de ordem ambiental (Muro-Reyes et al., 2011) e resulta em menos gastos com alimentação (Arthur e Herd, 2008).

O consumo alimentar residual (CAR) é uma medida para mensuração da eficiência alimentar dos animais e é definido como a diferença entre o consumo de matéria seca observado $\left(\mathrm{CMS}_{\mathrm{obs}}\right)$ e o consumo estimado $\left(\mathrm{CMS}_{\mathrm{est}}\right)$, conforme proposto por Koch et al. (1963) para bovinos. Um valor mais positivo de CAR denota que o animal apresenta consumo observado maior do que o esperado para determinado nível de produção, ou seja, é menos eficiente. Em contrapartida, quanto menor o valor do CAR, mais representativa é a diferença entre o consumo predito e o observado de um animal, portanto este aproveita mais eficientemente o alimento para ganho de peso.

A importância do CAR como ferramenta para identificação e seleção de animais de produção mais eficientes tem sido destacada por diversos autores, principalmente pelo fato da ausência de relações dessa característica com medidas de crescimento, tais como o peso vivo e a taxa de ganho do animal (Arthur et al., 2001a; Herd et al., 2003; Schenkel et al., 2004). Todavia, de acordo com revisão realizada por Lanna e Almeida (2004) para bovinos de corte, diversos autores estimaram correlação positiva entre CAR e deposição de gordura subcutânea. Com isso, a seleção pelo CAR poderia ocasionar redução na deposição de gordura, o que prejudicaria o acabamento e a qualidade final das carcaças.

Ressalta-se que a grande maioria dos trabalhos disponíveis na literatura foi realizada com bovinos, sendo ainda escassos os resultados publicados para a espécie ovina. Assim sendo, o objetivo com a realização deste trabalho foi verificar as relações entre CAR, medidas de desempenho e eficiência animal e avaliações realizadas na carcaça por ultrassonografia em cordeiros da raça Ile de France. Também, buscou-se avaliar as diferenças relacionadas a essas características em animais classificados com base no CAR (alto ou baixo).

\section{MATERIAL E MÉTODOS}

O experimento foi realizado no Laboratório de Produção e Pesquisa em Ovinos e Caprinos da Universidade Federal do Paraná (LAPOCUFPR), situado na Fazenda Experimental do Canguiri, em Pinhais - PR, região metropolitana de Curitiba, entre os meses de setembro/2010 e novembro/2010, com aprovação (protocolo $\mathrm{n}^{\mathbf{0}}$ 010/2011) da Comissão de Ética no Uso de Animais do Setor de Ciências Agrárias da Universidade Federal do Paraná (CEUSCAUFPR).

Após 17 dias de adaptação ao manejo e à alimentação, 20 cordeiros machos da raça Ile de France com $115 \pm 8$ dias de idade e $31,3 \pm 4,1 \mathrm{~kg}$ de peso vivo inicial (PVI), filhos de mesmo pai, procedentes da Fazenda Tangará em Reserva $\mathrm{PR}$, na qual eram mantidos em pastagem, foram confinados durante 65 dias em baias individuais para determinação do consumo individual de alimento. As baias possuíam dimensões de aproximadamente $1,0 \mathrm{~m} \times 2,2 \mathrm{~m}$ e continham cochos individuais para ração e bebedouros.

A alimentação foi fornecida ad libitum, na forma de ração total misturada (Tab. 1) com a proporção volumoso:concentrado de 30:70, sendo ofertada três vezes ao dia, às oito, 13 e 17 horas. $\mathrm{O}$ arraçoamento diário era feito mantendose porcentagem de sobras de $10 \%$ do fornecido. A composição bromatológica média da ração (Tab. 1) foi obtida com a formação de amostras compostas semanais, analisadas no Laboratório de Nutrição Animal da UFPR, conforme Silva e Queiroz (2002) e, para os teores de fibra em detergente neutro e de fibra em detergente ácido, segundo Van Soest et al. (1991). Os valores dos nutrientes digestíveis totais (NDT) foram estimados pela fórmula de Weiss et al. (1992). A dieta atingiu o valor mínimo de 2,4Mcal EM/kg de MS recomendado para não limitar a ingestão em testes de consumo em bovinos, de acordo com a Beef Improvement Federation (Beef, 2010). 
Diariamente, as sobras de alimento eram retiradas dos cochos, pesadas e também amostradas, permitindo-se a formação de amostras compostas semanais para determinação do teor de MS das sobras, que foi, em média, de $76,2 \%$ no período.

Para obtenção do ganho de peso médio diário (GMD), que foi calculado por regressão linear entre o tempo de experimento e os pesos vivos (PV) individuais, foram realizadas pesagens a cada 13 dias, em condição de jejum de 12 horas. A primeira pesagem pós-adaptação ao confinamento individual foi representativa do PVI, e a última, aos 65 dias de experimento, representativa do peso vivo final (PVF).

Tabela 1. Ingredientes e composição bromatológica da ração total misturada fornecida aos cordeiros

\begin{tabular}{lc}
\hline Ingredientes & Inclusão $(\mathrm{g} / \mathrm{kg})$ \\
\hline Feno moído de azevém & 300,00 \\
Calcário calcítico & 11,90 \\
Casquinha de soja & 140,00 \\
Farelo de soja 44\% & 116,90 \\
Milho grão moído & 408,10 \\
Mistura mineral* & 23,10 \\
Monensina sódica & 0,024 \\
& \\
Composição química & $\%(\mathrm{MS})$ \\
Matéria seca & 87,70 \\
Proteína bruta & 14,12 \\
Extrato etéreo & 2,87 \\
Resíduo mineral & 6,32 \\
Fibra em detergente ácido & 18,14 \\
Fibra em detergente neutro & 34,63 \\
Nutrientes digestíveis totais & 66,56 \\
Cálcio & 1,13 \\
Fósforo & 0,47
\end{tabular}

*Ovinofós Núcleo Produção® - Tortuga Companhia Zootécnica Agrária. Níveis de garantia (por $\mathrm{kg}$ do produto) - P: 61g; Ca: 267g; S: 35g; Co: 20mg; Mn: 2000mg; Cu: 350mg; F: 610mg; Se: 23mg; Mg: 20g; Cr: 60mg; Mo: 500mg; Zn: 6000mg; Fe: 3000mg; I: $80 \mathrm{mg}$.

$\mathrm{O} \mathrm{CMS}_{\text {obs }}$ foi obtido pela média da subtração da quantidade de alimento diário fornecido pela quantidade de sobras diárias (ambos ajustados para MS). O $\mathrm{CMS}_{\mathrm{est}}$ foi modelado com auxílio do programa estatístico $\mathrm{R}$ Development Core Team (2010), por regressão do $\mathrm{CMS}_{\text {obs }}$ em função do peso metabólico $\left(\mathrm{PM}=\mathrm{PV}\right.$ édio $\left.^{0,75}\right)$ e do GMD de cada animal durante o período, conforme a equação $\left(\mathrm{R}^{2}=0,34\right): \mathrm{CMS}_{\mathrm{est}}=$ $0,09830+0,07470 * \mathrm{PV}_{\text {met }}+0,62617 * \mathrm{GMD}$. Utilizando-se a metodologia proposta por Koch et al. (1963), o CAR de cada animal foi obtido da diferença do $\mathrm{CMS}_{\mathrm{obs}}$ pelo $\mathrm{CMS}_{\text {est }}$.

A conversão alimentar foi calculada como a relação direta do $\mathrm{CMS}_{\mathrm{obs}}$ diário pelo GMD, enquanto a taxa de crescimento relativa foi obtida pela fórmula $\mathrm{TCR}=100 *(\log \mathrm{PVF}-$ $\log \mathrm{PVI}) / \mathrm{n}^{\mathbf{0}}$ de dias em experimento, de acordo com Fitzhugh e Taylor (1971). Para a taxa de Kleiber, considerou-se a divisão do GMD pelo PV metabólico (Kleiber, 1947).

Nos dias 32 e 65 do período experimental, foram feitas avaliações de carcaça por meio de imagens de ultrassom (Aloka Prosound 2®), com determinação das seguintes características: área de olho de lombo, profundidade do músculo Longissimus e espessura de gordura subcutânea entre a $12^{\text {a }}$ e $13^{\text {a }}$ costelas. Também foram avaliados os ganhos dessas características no decorrer do tempo, subtraindo-se os valores da primeira avaliação dos valores da segunda avaliação.

Para efeito de comparação, estabeleceram-se duas classes de eficiência entre os animais, baseadas no CAR: os menos eficientes (CAR alto: $0,5 * \mathrm{DP}$ acima da média, totalizando oito animais) e os mais eficientes (CAR baixo: $0,5 * \mathrm{DP}$ abaixo da média, totalizando seis animais).

Foram estimadas as correlações simples de Pearson utilizando-se o procedimento analítico cor.test do programa estatístico R Development Core Team (2010), e os dados das diferentes classes de eficiência foram submetidos à analise de variância para se verificarem as diferenças pelo teste $\mathrm{F}$ a $5 \%$ de significância.

\section{RESULTADOS E DISCUSSÃO}

O CAR se mostrou positivamente correlacionado $(\mathrm{P}<0,01)$ com o $\mathrm{CMS}_{\mathrm{obs}}$, o CMS (\%PV) e com a CA (Tab. 2). Semelhantemente, Snowder e Van Vleck (2003) estimaram correlação de 0,64 entre o CAR e o consumo de MS, enquanto Knott et al. (2010), Redden et al. (2011) e Muro-Reyes et al. (2011) encontraram coeficientes de, respectivamente, $0,68,0,77$ e 0,58 também para 
ovinos. Esses mesmos autores não encontraram correlações significativas entre CAR e GMD, corroborando os resultados aqui apresentados. $\mathrm{O}$ $\mathrm{CMS}_{\text {obs }}$ também apresentou correlação com a CA $\left(r_{\mathrm{CA}}=0,61\right)$.

O sentido e a dimensão dessas correlações confirmam a capacidade do CAR para identificação de animais mais eficientes, o que pode ser visto também pelas diferenças mostradas entre as classes, em que o grupo dos mais eficientes (CAR baixo) apresentou menor $(\mathrm{P}<0,05)$ ingestão de alimento, tanto no consumo total $(0,211 \mathrm{~kg}$ de $\mathrm{MS} / \mathrm{dia}$ a menos ou $12,92 \%$ inferior) quanto em $\%$ do $\mathrm{PV}$, para mesma faixa de ganho de peso $(\mathrm{P}>0,05)$. Entre as classes, houve diferença no CAR de $0,240 \mathrm{~kg}$ de MS/dia $(\mathrm{P}<0,01)$.

Tabela 2. Coeficientes de correlação de Pearson entre as medidas de desempenho e eficiência e o consumo alimentar residual (CAR) e a conversão alimentar (CA); e médias Łdesvios-padrão obtidos para essas características em cordeiros com alto ou baixo CAR

\begin{tabular}{|c|c|c|c|c|c|}
\hline \multirow[b]{2}{*}{ Variáveis } & \multicolumn{2}{|c|}{ Correlações } & \multicolumn{2}{|l|}{ CAR (classes) } & \multirow[t]{2}{*}{$\mathrm{P}$} \\
\hline & $r_{\text {CAR }}$ & $\mathrm{r}_{\mathrm{CA}}$ & $\begin{array}{c}\text { Alto } \\
(\mathrm{n}=8)\end{array}$ & $\begin{array}{l}\text { Baixo } \\
(n=6)\end{array}$ & \\
\hline $\mathrm{CMS}_{\mathrm{obs}}$ & $0,81 * *$ & $0,61 * *$ & $1,633 \pm 0,120 \mathrm{a}$ & $1,422 \pm 0,169 b$ & 0,02 \\
\hline CMS (\%PV) & $0,90 * *$ & 0,33 & $3,94 \pm 0,12 \mathrm{a}$ & $3,31 \pm 0,36 b$ & 0,01 \\
\hline GMD (kg/dia) & 0,00 & $-0,63 * *$ & $0,335 \pm 0,040$ & $0,329 \pm 0,031$ & 0,76 \\
\hline PVI & 0,01 & $0,51 * *$ & $31,250 \pm 4,314$ & $32,833 \pm 4,271$ & 0,51 \\
\hline PVF & $-0,01$ & 0,15 & $51,844 \pm 4,245$ & $53,208 \pm 3,706$ & 0,54 \\
\hline PM & $-0,00$ & 0,34 & $16,352 \pm 1,210$ & $16,787 \pm 1,141$ & 0,51 \\
\hline CA & $0,63 * *$ & - & $4,935 \pm 0,696$ & $4,359 \pm 0,702$ & 0,15 \\
\hline TCR & $-0,03$ & $-074 * *$ & $0,342 \pm 0,054$ & $0,326 \pm 0,047$ & 0,56 \\
\hline TK & 0,02 & $-0,75^{* *}$ & $0,021 \pm 0,003$ & $0,020 \pm 0,002$ & 0,41 \\
\hline CAR & - & $0,63 * *$ & $0,133 \pm 0,011 \mathrm{a}$ & $-0,137 \pm 0,148 b$ & 0,01 \\
\hline
\end{tabular}

$\mathrm{CMS}_{\mathrm{obs}}=$ Consumo de MS observado (kg de MS/dia); GMD = ganho médio diário; $\mathrm{PVI}=$ peso vivo inicial; $\mathrm{PVF}=$ peso vivo final; $\mathrm{PM}=$ peso metabólico médio; $\mathrm{CA}=$ conversão alimentar; $\mathrm{TCR}=$ taxa de crescimento relativo; $\mathrm{TK}=$ taxa de Kleiber; $* \mathrm{P}<0,05$ e $* * \mathrm{P}<0,01$. Letras distintas na mesma linha diferem pelo teste $\mathrm{F}(\mathrm{P}<0,05)$.

O coeficiente de correlação entre CAR e CA foi significativo $(\mathrm{r}=0,63, \mathrm{P}<0,01)$ e maior que os descritos por Arthur et al. (2001a), Lancaster et al. (2009) e Kelly et al. (2010) para bovinos em crescimento de $0,53,0,59$ e 0,46 , respectivamente, porém inferior ao estimado para cordeiros $\left(\mathrm{r}_{\mathrm{CAR}}=0,73\right)$ por Knott et al. $(2010)$. Seria importante verificar a existência de correlação genética de mesmo sentido entre tais parâmetros para ovinos, assim como encontrado em bovinos (Lancaster et al., 2009), pois, dessa forma, uma seleção para CAR melhoraria também a conversão alimentar dos animais. Apesar da correlação moderada entre CAR e CA no presente estudo, não houve diferença entre as classes de eficiência para este último componente.
As demais variáveis de desempenho e eficiência não se mostraram correlacionadas com o CAR $(\mathrm{P}>0,05)$, e animais de alto e baixo CAR apresentaram similaridade nesses itens. Quanto ao GMD, era esperado que não houvesse resultados significativos, pois a própria definição do CAR comporta o GMD no ajuste da regressão, o que leva à independência fenotípica entre essas características (Arthur et al., 2001a; Sobrinho et al., 2011). Em relação ao PVI, PVF e PM, os resultados aqui obtidos estão em concordância com os relatos de que o CAR não está ligado às medidas de tamanho corporal, como previamente descrito por Arthur et al. (2001b) e Carstens et al. (2002). Para TCR e TK, semelhante ao encontrado por Nkrumah et al. (2004) em bovinos de corte, também houve ausência de relações com o CAR de cordeiros. Uma vez que o cálculo de tais taxas leva em 
consideração os pesos vivos e o ganho de peso dos animais, e estes se mostraram similares neste experimento, justifica-se a ausência de significância para essas medidas.

Coeficientes de correlação significativos e negativos foram encontrados entre CA e GMD; CA e TCR; e CA e TK $\left(\mathrm{r}_{\mathrm{CA}}=-0,63, \mathrm{r}_{\mathrm{CA}}=-0,74\right.$ e $r_{C A}=-0,75$, respectivamente), enquanto entre CA e PVI foi positivo $\left(\mathrm{r}_{\mathrm{CA}}=0,51\right)$. Nkrumah et al. (2004) descreveram correlações muito parecidas entre CA e GMD, TCR e TK para bovinos mestiços, com coeficientes de, respectivamente, $0,63,-0,75$ e - 0,73 . Para animais Nelore, Santana (2009) estimou correlações de $-0,85$ para CA e GMD, e de 0,50 entre CA e PVI, este último muito próximo ao exposto para cordeiros neste estudo. No caso de bovinos, discutem-se muito os prejuízos da utilização da CA como critério de seleção, devido às relações com características de crescimento e tamanho corporal, que levariam à produção de animais maiores e com maior exigência nutricional de mantença (Arthur e Herd, 2008). Dessa forma, destaca-se, com base nos resultados deste trabalho, que existe a necessidade de se atentar para tal fato também na seleção de ovinos, especialmente em raças de maior porte e mais tardias. Adicionalmente, Cabral et al. (2008) discutiram a importância de se considerar eficiência alimentar em conjunto com medidas de desempenho na seleção de raças para produção de carne, devido à relação entre CA e tais medidas, buscando assim, reduzir o custo de manutenção desses animais na ovinocultura.

O CAR e a CA não se mostraram correlacionados $(\mathrm{P}>0,05)$ com nenhuma das características de carcaça avaliadas por ultrassom, e, da mesma forma, não houve diferença $(\mathrm{P}>0,05)$ entre as classes de eficiência alimentar pelo CAR para as variáveis analisadas (Tab. 3).

Tabela 3. Coeficientes de correlação de Pearson das medidas de carcaça avaliadas por ultrassom com o consumo alimentar residual (CAR) e a conversão alimentar (CA), e médias \pm desvios-padrão obtidos para essas características em cordeiros com alto ou baixo CAR

\begin{tabular}{|c|c|c|c|c|c|}
\hline \multirow[b]{2}{*}{ Variáveis } & \multicolumn{2}{|c|}{ Correlações } & \multicolumn{2}{|c|}{ CAR (classes) } & \multirow[b]{2}{*}{$\mathrm{P}$} \\
\hline & $\mathrm{r}_{\mathrm{CAR}}$ & $\mathrm{r}_{\mathrm{CA}}$ & $\begin{array}{c}\text { Alto } \\
(\mathrm{n}=8)\end{array}$ & $\begin{array}{l}\text { Baixo } \\
(n=6)\end{array}$ & \\
\hline AOL intermediária $\left(\mathrm{cm}^{2}\right)$ & 0,09 & 0,36 & $11,74 \pm 1,23$ & $12,09 \pm 1,37$ & 0,62 \\
\hline AOL final $\left(\mathrm{cm}^{2}\right)$ & 0,19 & 0,16 & $14,40 \pm 1,13$ & $14,39 \pm 1,79$ & 0,99 \\
\hline AOL ganho $\left(\mathrm{cm}^{2}\right)$ & 0,11 & $-0,14$ & $2,66 \pm 1,24$ & $2,29 \pm 1,57$ & 0,63 \\
\hline EGS intermediária $(\mathrm{cm})$ & $-0,30$ & 0,29 & $0,45 \pm 0,09$ & $0,52 \pm 0,08$ & 0,16 \\
\hline EGS final $(\mathrm{cm})$ & $-0,04$ & 0,24 & $0,58 \pm 0,11$ & $0,59 \pm 0,07$ & 0,79 \\
\hline EGS ganho $(\mathrm{cm})$ & 0,25 & $-0,02$ & $0,12 \pm 0,09$ & $0,07 \pm 0,05$ & 0,21 \\
\hline PROF. intermediária $(\mathrm{cm})$ & 0,14 & 0,39 & $2,36 \pm 0,16$ & $2,38 \pm 0,24$ & 0,87 \\
\hline PROF. final $(\mathrm{cm})$ & 0,07 & 0,10 & $2,77 \pm 0,21$ & $2,82 \pm 0,31$ & 0,73 \\
\hline PROF. ganho (cm) & $-0,05$ & $-0,22$ & $0,41 \pm 0,23$ & $0,44 \pm 0,27$ & 0,82 \\
\hline
\end{tabular}

$\mathrm{AOL}=$ área de olho de lombo, EGS = espessura de gordura subcutânea, PROF. = profundidade do músculo Longissimus; $* \mathrm{P}<0,05$ e ** $\mathrm{P}<0,01$. Letras distintas na mesma linha diferem pelo teste $\mathrm{F}(\mathrm{P}<0,05)$.

Basarab et al. (2003) e Lancaster et al. (2009) estimaram correlações significativas entre CAR e espessura de gordura subcutânea em bovinos de raças europeias, e para bovinos da raça Nelore, Leme e Gomes (2007) relataram também menor espessura de gordura em animais de CAR baixo, ou seja, os mais eficientes. Knott et al. (2003), em trabalho com ovinos, encontraram correlação negativa entre carcaça magra e CAR. Todos esses relatos sugerem que animais mais eficientes pelo CAR tendem a apresentar menos gordura na carcaça.
Isso não foi confirmado, entretanto, pelos resultados obtidos no presente estudo, embora os cordeiros mais eficientes tenham apresentado maiores níveis séricos de creatinina (Paula et al., 2011) e esse metabólito seja frequentemente associado com alterações na composição corporal e no padrão de deposição de gordura (Richardson et al., 2004). A ausência de significância pode ser atribuída à idade e maturidade reduzidas dos animais experimentais (aproximadamente 3,8 a 6 meses). De acordo com Prescott (1982), o crescimento do cordeiro é descrito como uma curva sigmoide, com 
aceleração da velocidade até que a puberdade seja atingida e diminuição gradativa até $o$ alcance da maturidade. Santos et al. (2001) ressaltaram ainda que os diferentes componentes da carcaça (ossos, músculo e gordura) apresentam padrões distintos de desenvolvimento, sendo que os músculos têm crescimento mais acelerado em cordeiros mais jovens, e a gordura em animais mais maduros, enquanto os ossos crescem mais lentamente que os demais constituintes. Portanto, devido ao fato de os cordeiros estarem em fase menos avançada na curva de crescimento, a proporção de gordura na composição do ganho corporal pode não ter sido representativa o suficiente para permitir que fossem detectados resultados significativos na idade utilizada neste experimento. Vale lembrar que a puberdade em ovinos se manifesta entre seis e nove meses de idade (Price et al., 1995). Dessa maneira, sugere-se a realização de pesquisas similares com ovinos em idade mais avançada.

\section{CONCLUSÕES}

Nas condições deste experimento, foi possível confirmar o potencial de utilização do CAR como medida de eficiência alimentar para cordeiros em confinamento, sem existência de relações com o ganho de peso e o tamanho corporal dos animais. Ainda, o CAR de cordeiros não apresentou associações com alterações nas características in vivo da carcaça.

\section{AGRADECIMENTOS}

Aos Srs. Júlio Pisani e Júlio Pisani Filho, proprietários da Fazenda Tangará, por cederem os animais para este experimento.

\section{REFERÊNCIAS}

ARTHUR, P.F.; ARCHER, J.A.; JOHNSTON, D.J. et al. Genetic and phenotypic variance and covariance components for feed intake, feed efficiency, and other postweaning traits in Angus cattle. J. Anim. Sci., v.79, p.2805-2811, 2001a.

ARTHUR, P.F.; RENAND, G.; KRAUSS, D. Genetic and phenotypic relationships among different measures of growth and feed efficiency in young Charolais bulls. Livest. Prod. Sci., v.68, p.131-139, 2001b.
ARTHUR, P.F.; HERD, R.M. Residual feed intake in beef cattle. Rev. Bras. Zootec., v.37, p.269-279, 2008.

BASARAB, J.A.; PRICE, M.A.; AALHUS, J.L. et al. Residual feed intake and body composition in young growing cattle. Can. J. Anim. Sci., v.83, p.189-204, 2003.

BEEF improvement federation - BIF. Guidelines for uniform beef improvement programs. Raleigh: U.S. Dept. Agriculture - North Carolina State University, 2010.

CABRAL, L.S.; SANTOS, J.W.; ZERVOUDAKIS, J.T. et al. Consumo e eficiência alimentar em cordeiros confinados. Rev. Bras. Saúde e Prod. Anim., v.9, p.703-714, 2008

CARSTENS, G.E.; THEIS, C.M.; WHITE, M.B. et al. Relationships between net feed intake and ultrasound measures of carcass composition in growing beef steers. Beef Cattle Res. in Texas, p.31-34, 2002.

FITZHUGH, H.A.; TAYLOR, C.S.St. Genetic analysis of degree of maturity. J. Anim. Sci., v.33, p.717-725, 1971.

FOOD and Agriculture Organization of the United Nations - FAO. FAOSTAT, 2009. Disponível em: <http://faostat.fao.org/>. Acessado em: 2 out. 2011.

HERD, R.M.; ARCHER, J.A.; ARTHUR, P.F. Reducing the cost of beef production through genetic improvement in residual feed intake: Opportunity and challenges to application. J. Anim. Sci., v.81, p.9-17, 2003.

KELLY, A.K.; MCGEE, M.; CREWS, JR. et al. Effect of divergence in residual feed intake on feeding behavior, blood metabolic variables, and body composition traits in growing beef heifers. J. Anim. Sci., v.88, p.109-123, 2010.

KLEIBER, M. Body size and metabolic rate. Physiol. Rev., v.27, p.511-541, 1947.

KNOTT, S.A.; CUMMIINS, L.J.; DUNSHEA, F.R. et al. Feed efficiency and body composition are related to cortisol response to adrenocorticotropin hormone and insulin-induced hypoglycemia in rams. Dom. Anim. Endocr., v.39, p.137-146, 2010.

KNOTT, S.A.; LEURY, B.J.; CUMMINS, L.J. et al. Relationship between body composition, net feed intake and gross feed conversion efficiency in composite sire line sheep. In: 'EAAP Publication, n.109, 2003. p.525-528.

KOCH, R.M.; SWIGER, L.A.; CHAMBERS, D. et al. Efficiency of feed use in beef cattle. J. Anim. Sci., v.22, p.486-494, 1963. 
LANCASTER, P.A.; CARSTENS, G.E.; CREWS, JR., D.H. et al. Phenotypic and genetic relationships of residual feed intake with performance and ultrasound carcass traits in Brangus heifers. J. Anim. Sci., v.87, p.3887-3896, 2009.

LANNA, D.P.D.; ALMEIDA, R. Exigências nutricionais e melhoramento genético para eficiência alimentar: experiências e lições para um projeto nacional. In: REUNIÃO ANUAL DA SOCIEDADE BRASILEIRA DE ZOOTECNIA, 41., 2004, Campo Grande. Anais ... Campo Grande: SBZ, 2004. p.248259.

LEME, P.R.; GOMES, R.C. Características de carcaça de novilhos Nelore com diferente consumo alimentar residual. In: REUNIÓN ASOCIASÓN LATINOAMERICANA DE PRODUCION ANIMAL, 20., 2007, Cuzco. Anais ... Cuzco: ALPA, 2007. (Resumo).

MURO-REYES, A.; GUTIERREZ-BANUELOS, H.; DIAZ-GARCIA, L.H. et al. Potential Environmental Benefits of Residual Feed Intake as Strategy to Mitigate Methane Emissions in Sheep. J. Anim. Vet. $A d v$. v.10, p.1551-1556, 2011.

NKRUMAH, J.D.; BASARAB, J.A.; PRICE, M.A. et $a l$. Different measures of energetic efficiency and their phenotypic relationships with growth, feed intake, and ultrasound and carcass merit in hybrid cattle. J. Anim. Sci., v.82, p.2451-2459, 2004.

PAULA, E.F.E.; SANTANA, M.H.A.; MONTEIRO, A.LG. et al. Consumo alimentar residual e o perfil metabólico sanguíneo de cordeiros Ile de France. In CONGRESSO BRASILEIRO DE ZOOTECNIA, 21., 2011, Maceió. Anais ... Maceió: Zootec, 2011, CDROM (Resumo).

PRESCOTT, J.H.D. Crecimiento y desarrollo de los corderos. In: HAPEZ, E.S.E. (Ed). Crescimento $e$ desarollo de los corderos. Zaragoza: Acribia. 1982, p.35-369.

PRICE, E.O.; BORGWARDT, R.; DALLY, M.R. Heterosexual experience differentially affects the expression of sexual behavior in 6- and 8-month-old ram lambs. Appl. Anim. Behav. Sci., v.46, p.193-199, 1995.

R DEVELOPMENT CORE TEAM. $R$ : A language and environment for statistical computing. $\mathrm{R}$ Foundation for Statistical Computing, Vienna, Austria. 2010, ISBN 3-900051-07-0, URL: http://www.Rproject.org
REDDEN, R.R.; SURBER, L.M.M.; ROEDER, B.L. et al. Residual feed efficiency established in a postweaning growth test may not result in more efficient ewes on the range. Small Rumin. Res., v.96, p.155159,2011

RICHARDSON, E.C.; HERD, R.M.; ARCHER J.A. et al. Metabolic differences in Angus steers divergently selected for residual feed intake. Aust. J. Exp. Agric., v.44, p.441-452, 2004.

SANTANA, M.H.A. Relação do consumo alimentar residual e conversão alimentar com características de carcaça, perfil metabólico e sanguíneo de touros da raça nelore. 2009. 75f. Dissertação (Mestrado em Ciências Veterinárias) - Universidade Federal do Paraná, Curitiba.

SANTOS, C.L.; PÉREZ, J.R.O.; MUNIZ, J.A. et al. Desenvolvimento relativo dos tecidos ósseo, muscular e adiposo dos cortes da carcaça de cordeiros Santa Inês. Rev. Bras. Zootec., v.30, p.487-492, 2001.

SCHENKEL, F.S.; MILLER, S.P.; WILTON, J.W. Genetic parameters and breed differences for feed efficiency, growth, and body composition traits of young beef bulls. Can. J. Anim. Sci., v.84, p.177-185, 2004.

SILVA, D.J.; QUEIROZ, A.C. Análise de alimentos: métodos químicos e biológicos. Viçosa, MG: Universidade Federal de Viçosa, 2002. 235p.

SOBRINHO, T.L.; BRANCO, R.H.; BONILHA, S.F.M. et al. Residual feed intake and relationships with performance of Nellore cattle selected for post weaning weight. Rev. Bras. Zootec., v.40, p.929-937, 2011.

SNOWDER, G.D.; VAN VLECK, L.D. Estimates of genetic parameters and selection strategies to improve the economic efficiency of postweaning growth in lambs. J. Anim. Sci., v.81, p.2704-2713, 2003.

VAN SOEST, P.J.; ROBERTSON, J.B.; LEWIS, B.A. Methods for dietary fiber, neutral detergent fiber, and no starch polysaccharides in relation to animal nutrition. J. Dairy Sci., v.74, p.3583-3597, 1991.

WEISS, W.P.; CONRAD, H.R.; ST. PIERRE, N.R. A theoretically based model for predicting total digestible nutrient values of forages and concentrates. Anim. Feed Sci. Technol., v.39, p.95-110, 1992. 